\title{
THE DEEP PROJECT
}

\author{
DAVID C. KOO \\ UCO/Lick Observatory, University of California, \\ Santa Cruz, CA 95064, USA
}

\begin{abstract}
.
DEEP is a multi-institutional program designed to undertake a major new spectroscopic survey of faint field galaxies with the Keck II 10-m telescope. The scientific goals are broad and include exploring galaxy formation and evolution, mapping the large scale structure at moderate to high redshifts, and constraining the nature and distribution of dark matter and cosmology. Besides the primary goal of securing large numbers of redshifts $\left(10,000^{+}\right)$to very faint limits of $I \sim 23$, DEEP intends to acquire spectra of high enough quality and spectral resolution to extract rotation curves, velocity dispersions, age estimates, and chemical abundances for a brighter subset of galaxies. A new imaging spectrograph for Keck called DEIMOS has been specifically designed to achieve these goals and is currently scheduled for completion by the end of 1998. DEIMOS will provide an overall gain for multi-object spectroscopy of about $7 \mathrm{x}$ compared to the current low-resolution spectrograph (LRIS). While awaiting for DEIMOS to be operational, the interim DEEP science programs have been diverse, but largely concentrated on spectroscopy of faint galaxies observed with HST, especially in the "Groth Strip" and Hubble Deep Field (HDF) and its flanking fields. Recent highlights include redshift and kinematic studies of compact galaxies, high redshift $(z \sim 3)$ galaxies, and distant spirals.
\end{abstract}

\section{Introduction}

As we approach the 21st century, the suite of new 8-10 m class optical telescopes promises to offer spectroscopic surveys of galaxies to unprecedented faintness. Equally tantalizing are the new opportunities to combine such deep ground-based spectra with deep space-based measurements of high spatial resolution, such as size, shape, and morphology, from the Hubble Space Telescope (HST), as well as in new wavebands, such as can be expected from SIRTF and AXAF. Besides supplying redshifts that are so critical to convert, e.g., magnitudes to intrinsic luminosities, and to obtain the lookback time, the high $\mathrm{S} / \mathrm{N}$ and high spectral-resolution from 8-10 m class telescopes provide three new, powerful diagnostics for the analysis of distant galaxies, namely internal velocities (and hence masses when size is used), abundances, and age estimates. These parameters are clearly important and independent probes of galaxies in the early universe and provide via mass, e.g., more direct links to the outputs of cosmological simulations of galaxy formation and evolution. Finally, because both galaxy evolution and their large scale patterns are very likely to be complex, surveys with large samples will be needed to address these problems.

\section{What is DEEP?}

To meet the aforementioned challenge, the Deep Extragalactic Evolutionary Probe (DEEP: more details at website http://www.ucolick.org/ deep/home.html) has been initiated as a project designed to gather spectral data for over 10,000 faint field galaxies (Mould 1993, Koo 1995) using the Keck II 10-m telescope and a new instrument (DEIMOS). Depths are expected to be $I \sim 23$ or fainter, corresponding to median redshifts $z \sim 0.8$. DEEP will thus be at least $10 \mathrm{x}$ larger and one magnitude deeper than the pioneering and important Canada France Redshift Survey (CFRS: sec proceedings). Based on our experience with Keck so far, redshifts are expected for 
the vast fraction of this sample $(\sim 80 \%$, which is comparable to the $75 \%$ reached in CFRS for $21<I<22$ ). The redshifts will be used not only to study the galaxies themselves, but also to map the clustering patterns and global velocity distortions and motions of distant galaxies. Since the large-scale structure and its evolution to redshifts $z \sim 1$ are quite sensitive to the amount and nature of dark matter as well as cosmology (Jenkins et al. 1997), DEEP is expected to place powerful independent constraints on both.

A distinguishing aspect of DEEP is that the survey also aims to gather internal kinematic data in the form of rotation curves or linewidths. Such measurements are of special interest because, if the system is bound and if sizes and inclinations can be independently measured or estimated, the inferred gravitational potential depth can be used to estimate the actual masses of distant galaxies. Tracking how the volume density of galaxies with different masses (as well as luminosities, sizes, structure, etc.) vary with lookback time will provide an entirely new dimension to study the rate of galaxy merging, luminosity evolution, disk and bulge structure formation, etc. Finally, if the mass, luminosity, and number evolution of galaxies can be better understood, the large sample of DEEP will provide an unprecedented opportunity to measure the geometry of the universe not only through the evolution of large scale structure but also via the classical volume test. Such volume tests are more sensitive than size or luminosity tests by factors of $\sim(1+z)^{2}$ and $\sim(1+z)$, respectively.

\section{What is DEIMOS?}

DEIMOS (DEep Imaging Multi-Object Spectrograph) is a new spectrograph for Keck II (more information is at website http://www.ucolick.org/ loen/Deimos/deimos.html). DEIMOS will provide both a wide-field direct imaging capability as well as multi-object spectroscopy via the use of focal-plane masks with multiple slitlets. Besides being optimal for DEEP, this Nasymth instrument will be ideal to tackle a broad range of targets that are densely populated in a small region of sky, including dense star fields, stars in globular clusters, extragalactic globular clusters, clusters of galaxies, etc. It is expected to be commissioned by late 1998 and operational by 1999.

Although DEIMOS was originally planned to be a four camera system, and then a two camera system, DEIMOS is currently being constructed with only one camera. This camera will include a CCD mosaic of $8 \mathrm{~K} \times 8 \mathrm{~K}$ comprised of eight $2 \mathrm{~K} \times 4 \mathrm{~K}$ CCDs with pixels of $15 \mu$. Not only will this camera accommodate a total slit length of over 16 arcmin (or roughly 75 slitlits) and an imaging area of of roughly 76 square arcmin at 0.12 arcsec per pixel, but also it will allow the spectra to span a large range in a single exposure with moderately high spectral resolution. The current design includes a low resolution mode with a $600 \mathrm{1} / \mathrm{mm}$ grating that provides $0.65 \AA$ per pixel over, e.g., the nominal range of $4300 \AA$ to $9500 \AA$ and a high resolution mode with a 1200 $1 / \mathrm{mm}$ grating that provides $0.33 \AA$ per pixel over, e.g., $6800 \AA$ to $9400 \AA$. The wavelength useable for spectroscopy spans from $4300 \AA$ to $11000 \AA$ while the imaging mode will reach bluer limits and will have standard $B, V, R, I$, and two user filters. After including the silver-coated Keck II telescope, the end-to-end throughput is expected to be $40 \%$ for imaging and nearly $30 \%$ for spectroscopy. Present plans provide for 10 pre-fabricated slit masks for each camera. Compared to Keck's current Low Resolution Imaging Spectrograph (LRIS: Oke et al. 1995), DEIMOS has a total throughput (sensitivity, spectral range, resolution, area) that is roughly $7 \mathrm{x}$ greater.

\section{Summary of Early DEEP Projects}

While waiting for the completion of DEIMOS so that the major DEEP survey of $10,000^{+}$galaxies can begin, we have undertaken a number of smaller, pilot-style projects with the existing LRIS to determine what is feasible with Keck and thus to help define the DEEP survey. To maximize the scientific returns for our relatively small samples, we have chosen to focus our efforts in fields where relatively deep HST WFPC2 images already exist. Such HST images are crucial not only for determining the morphology of our galaxy samples, but also for obtaining reliable measures of structure, including size and inclination, which are needed to convert kinematic observations from Keck into direct measures of mass. Overall, our early results have been encouraging and reassure us that our major DEIMOS survey is feasible. 

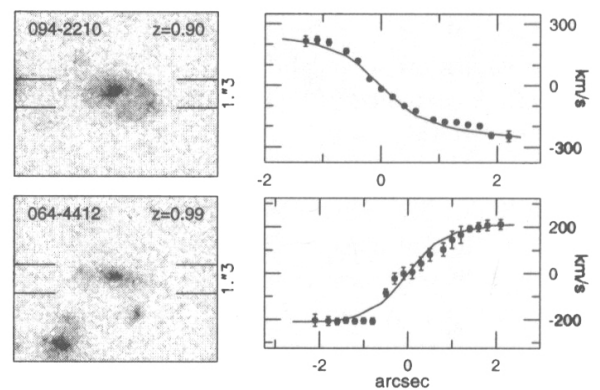

Figure 1. Fig. 1. Examples of the rotation curves measured for two high redshift galaxies (Vogt et al. 1996).

\subsection{SURVEY DEPTH AND COMPLETENESS}

Our faintest redshift samples are too small to obtain any detailed assessment of the expected completeness in securing reliable redshifts as a function of total $I$ band magnitudes, size, surface brightness, strength of emission lines, redshift, etc. Based on a representive sample of 33 galaxies that reached fainter than $I \sim 24$ in the Groth Strip, we find that our exposures of 1.5 to 3.5 hours yielded a completeness of $100 \%$ to the $I \sim 22$ limit of CFRS and over $80 \%$ for the entire sample (Koo et al. 1996). A large fraction of all successful redshifts greater than $z \sim 0.9$ are based on the detection of the [OII] doublet at $3727 \AA$, which can easily be hidden by the atmospheric " $\mathrm{A}$ " band absorption at $7600 \AA$ and the dense forest of strong night sky $\mathrm{OH}$ lines. New redshifts from our most recent observing runs indicate that our completeness is fully consistent with those found above, with over $80 \%$ completeness to $I \sim 23$. Thus the DEEP survey will be able to achieve such completeness for 1.5 to 2 hour exposures for roughly four sets of targets (i.e., about 300 total targets) for each clear night. Thus a program to target 20,000 galaxies with a final yield of 16,000 galaxies to $I \sim 23$ can be completed with roughly 70 clear nights. Assuming a $30 \%$ loss due to weather and other adverse conditions, a 100 night program would be needed for such a survey.

We note that these faint and relatively high redshifts also provide a crucial set of tests and calibration targets for the use of broadband photometry to estimate redshifts. Within our DEEP team, such photometric redshifts have been studied by Koo 1985; Connolly et al. 1995; Connolly et al. 1997; Brunner 1997 and shown to be one of the most promising and reliable techniques to push beyond the limits of spectroscopy. Such photometric redshifts, which can be estimated with relatively inexpensive multicolor images, are expected to play an important role in DEEP. As one example, we plan to use photometric redshifts to improve probes of mass that rely on weak lensing distortions. As another, we intend to maximize the number of galaxies from a given redshift interval in the selection of the DEEP sample used to track the evolution in large scale structures. A demonstration of the power of photometric redshifts for survey pre-selection is the use of $U$ band drop-outs to define the spectroscopic samples that led to the discovery of large scale structures at, redshifts $z \sim 3$ by Steidel et al (1997).

\subsection{ROTATION CURVES OF DISTANT SPIRALS}

One of the major themes of DEEP is the kinematics of distant galaxies. As seen in Fig. 1, we have clearly demonstrated the feasibility of discerning emission-line rotation curves of likely spirals seen to redshifts near $z \sim 1$ for galaxies as faint as $I \sim 22$ with one to two hour exposures. To ensure alignment with the apparent major axis, the slitlets have been milled with tilts. Based on a total sample of 16 galaxies, we find little evidence for any major change $(<0.6 \mathrm{mag})$ in the zero-point of the optical Tully-Fisher relation (Vogt et al. 1996; Vogt et al. 1997). The DEEP results are in stark contrast to claims for more extensive evolution of $1.5 \mathrm{mag}$ (Rix et al. 1997) to 2.5 mag (Simard and Pritchet 1997) for very blue galaxies. 


\subsection{EMISSION LINEWIDTHS}

Although rotation curve measures are possible for extended galaxies, the vast majority of very faint galaxies are in fact too small to yield more than linewidths as kinematic data. Except for very bright galaxies that yield absorption linewidths, emission lines are used. Though winds, dust obscuration, and poor representation of the gravitational potential by the luminous star formation regions may all invalidate the use of the emission line widths for probing the gravitational potential, especially for very compact, starbursting systems, the available data to date on such extreme galaxies show a fairly tight ratio of $0.7+/-0.1$ between such optical line-width measures and radio measures of HI motions (Telles and Terlevich 1993). The HI kinematics presumably sample most of the true potential.

We have thus proceeded with the assumption that linewidths, after an upward correction of $40 \%$, are meaningful measures of the true potential. Then when combined with HST size data, we are able to obtain masses. At least for blue compact galaxies, we find that luminosity alone can be a very poor gauge of their masses, i.e., the $\mathrm{M} / \mathrm{L}$ ratio can vary enormously (Guzmán et al. 1996). Indeed, for some very compact galaxies brighter than the Milky Way, we needed the the High Resolution Echelle Spectrograph (HIRES: see Vogt et al. 1994) to resolve velocity widths smaller than $30 \mathrm{~km}-\mathrm{s}^{-1}$ (Koo et al. 1995). Fortunately for the DEEP program, the vast majority of faint galaxies appear to have linewidths that can be resolved at the nominal DEIMOS spectral resolution of $\sigma \sim 40 \mathrm{~km}-\mathrm{s}^{-1}$. This was empirically verified for a sample of compact galaxies, and thus most likely to have the smallest masses (Phillips et al. 1997; Guzmán et al. 1997). The key point is that the lack of correlation between luminosity and mass, i.e. stable $\mathrm{M} / \mathrm{L}$, demonstrates the usefulness, necessity, and promise of kinematics as an important new dimension to discern the evolution of different galaxy populations.

\subsection{VERY HIGH REDSHIFT $Z \sim 3$ GALAXIES}

One of the major advances with Keck has been the dramatic demonstration by Steidel et al (1996a) that very high redshift $(z \sim 3)$ galaxies, originally selected on the basis of broadband colors, can be routinely confirmed spectroscopically. The DEEP team has extended the pioneering efforts of Steidel et al. (1996b) in the Hubble Deep Field (HDF) by pushing the faintness limits beyond $I \sim 25$, using redder "dropouts" to reach higher redshifts and higher levels of completeness, and adopting higher spectral resolutions to improve kinematic measurements (Lowenthal et al. 1997). Based on the evidence so far, we suggest that the high redshift galaxies may also be small mass systems that become dwarfs today or that later merge to form more massive galaxies instead of being only the cores of massive galaxies. Regardless of the eventual understanding of their nature, the success of these surveys now open another possible avenue for the DEEP survey with DEIMOS, namely an exploration of not only the $z \sim 1$ universe, but also the very high redshift regime with 1000 's of galaxies at $z \sim 3$ or beyond, where galaxy formation and pre-merger subcomponents are likely to be in their earliest phases.

\section{Summary and Future Plans}

Our various pilot programs with LRIS clearly show the need and power of kinematics for galaxy surveys, the feasibility of reaching $I \sim 23$ or fainter with respectable completeness in one to two hour exposures with Keck, and the accessiblity of the very high redshift universe. Thus the definition, feasibility, and value of the DEIMOS DEEP program is no longer merely in the speculative proposal stage. The selection of the targets has not yet been fully settled, but is likely to be dominated by the need for very large numbers of galaxies in the large scale structure program. Simulations to optimize the sample definition have not yet been completed, but the basic survey is anticipated to consist of redshift slices near $z \sim 1$, with the spectroscopic sample chosen from photometric redshifts. The final choice of fields for the DEIMOS large-scale structure survey has not yet been settled, but is very likely to be comprised of four or five regions of roughly 16 'x $120^{\prime}$ each and accessible from spring to fall. The final choices may be dictated by the availability of deep, wide-field multicolor images being collected by several different groups. 


\section{Acknowledgements}

The DEEP scientific program was initiated and enjoyed financial support (NSF AST 91-20005) from the Berkeley Center for Particle Astrophysics (CfPA), an NSF Science and Technology Center aimed to explore the nature of dark matter, as well as an NSF grant, AST 95-29098. The scientific DEEP program includes teams from several institutions, including a core group comprised of Garth Illingworth, S. M. Faber, and the author at UC Santa Cruz, Marc Davis at UC Berkeley, Richard Kron at the Univ. of Chicago/Fermi Lab, Alex Szalay at Johns Hopkins, and Chuck Steidel and Roger Blandford at Caltech. Except for management, the bulk of the hard work has been undertaken by a large pool of talented junior astronomers: Duncan Forbes (now at Birmingham), Rafael Guzmán (now at Yale), Jesus Gallego (U. of Madrid), James Lowenthal (now at U. of Mass., Amherst), Drew Phillips, Nicole Vogt, Luc Simard, and Vicki Sarajedini (all at UC Santa Cruz), Matthew Bershady (now U. of Wisconsin), Andy Connolly and Robert Brunner (Johns Hopkins), and Marianne Takamiya and Brad Holden (Univ. of Chicago).

The instrument (DEIMOS) is being funded largely by NSF through its Academic Research and Infrastructure Program and by the Keck foundation (CARA). The instrument effort is being led by S. M. Faber with support from Richard Stover (UC Santa Cruz) for the CCD effort; David Cowley who is Project Manager; and a team of dedicated technical experts and staff among the UC Observatories that is transforming design goals into a working instrument.

\section{References}

Brunner, R. J. 1997, Ph.D. thesis, Johns Hopkins Univ.

Connolly, A. J., et al. 1995, $A J, 110,2655$

Connolly, A. J., et al. 1997, $A p J, \mathbf{4 8 6}$, L11

Guzmán, R. et al. 1996, $A p J, \mathbf{4 6 0}$, L5

Guzmán, R. et al. $1997, A p J, 489,559$

Jenkins, A., et al. 1997, $A p J$, submitted (Astro-ph 9709010)

Koo, D. C. $1985, A J, \mathbf{9 0}, 418$

Koo, D. C. 1995, Wide Field Spectroscopy and the Distant Universe, eds. S. J. Maddox and A. Aragón-Salamanca, p. 55

Koo, D. C. et al. $1995, A p J, 440, \mathrm{~L} 49$

Koo, D. C. et al. $1996, A p J, \mathbf{4 6 9}, \mathbf{5 3 5}$

Lowenthal, J. D. et al. 1997, $A p J, 481,673$

Mould, J. 1993, ASP Conf. Ser., 43, 281

Oke, J. B. et al. 1995, PASP, 107, 375

Phillips, A. C. et al. $1997, A p J, \mathbf{4 8 9}, 543$

Rix, H.-W., et al. 1997, MNRAS, 285, 779

Simard, L., and Pritchet, C. $1997, A p J$, submitted

Steidel, C. C., et al. 1996a, $A J, \mathbf{1 1 2}, 352$

Steidel, C. C., et al. 1996b, ApJ, 462, L17

Steidel, C. C., et al. $1997, A p J$, in press

Telles, E., \& Terlevich, R. 1993, Ap\&SS, 205, 49

Vogt, N. P. et al. $1996, A p J, 465$, L15

Vogt, N. P. et al. 1997, $A p J, 479$, L121

Vogt, S., et al. 1994, Proc. SPIE, 2198, 362 